\title{
La competencia esencial
}

de la corporación*

\section{C.K. Prahalad y Gary Hame!}

7 odavía es desconocida para muchas compañias cuál es la maI nera más firme de prevalecer en la competencia global. Durante los ochenta, a los a los ejecutivos se les juzgó por su habilidad para reestructurar, reordenar y desestratificar sus corporaciones. En los noventa, serán juzgados por su habilidad para identificar, cultivar y explotar las capacidades esenciales que hacen posible el crecimiento. En verdad, ellos tendrán que repensar el concepto mismo de Corporación.

Considere los últimos 10 años de GTE y NEC. Al principio de los ochenta, la GTE estaba en la inmejorable posición de convertirse en el mejor competidor de la evolucionante industria de la tecnología de la información. Era activa en telecomunicaciones. Sus operaciones abarcaban una variedad de negocios que incluian teléfonos, sistemas de transmisión y de conmutación, PABX digitales, semi-

- Tomado de Harvard Business Review, May-June 1990. pp 79.89.

Traducido por Antonio Navarrefe Nielo conduclores, paquetes de conmutación, satélites, sistemas de defensa y productos de iluminación. Y el grupo de productos de entretención de GTE, que producía los televisores a color Sylvania, tenía una posición en tecnologias relacionadas de exhibición. En 1980. Las ventas de GTE fueron de $\$ 9.98$ billones, y el flujo de caja neto fue de $\$ 1.73$ billones. Por el contrario, NEC tuvo ventas mucho menores. Sólo $\$ 3.8$ billones. Tenía una base tecnológica similar y negocios de computadores, pero no tenía experiencia como compañía operativa de telecomunicaciones.

Sin embargo, mire las posiciones de GTE y NEC en 1988. Las ventas de GTE en 1988 fueron de $\$ 16.46$ billones y las ventas de NEC fueron considerablemente superiores: $\$ 21.89$ billones. GTE se ha convertido de hecho, en una compañia operativa de teléfonos con una posición en productos de iluminación y de defensa. En términos globales los demás negocios de GTE son pequeños. GTE se desvinculó de 
Sylvania T.V. y Telenet, se arriesgó colocando conjuntamente conmuta- ción, transmisión y PABX digitales y dejó de producir semiconductores. Como resultado, se ha desgastado la posición internacional de GTE. Ninguna renta de los EE.UU. en términos de porcentaje del ingreso total bajó de $20 \%$ al $15 \%$ entre 1980 y 1988.

NEC ha surgido como el líder mundial en semiconductores y como un competidor de primera línea en productos de telecomunicaciones y computadores. Ha consolidado su posición en computadores centrales. Ha sobrepasado la conmutación y transmisión públicas para incluir productos de moda cono teléfonos móviles, máquinas de facsímiles y computadores Laptop portátiles, estableciendo un puente entre las telecomunicaciones y la automatización de las oficinas. NEC es la única compañía en el mundo que está entre las cinco primeras en ingresos en telecomunicaciones, semiconductores y computadores cenrales. ¿Por qué estas dos compañías, que empezaron con similares portafolios de negocios, se desempeñaron en forma tan diferente? Principalmente porque NEC se vio a sí misma en términos de "Competencias esenciales" y GTE no.

\section{Repensar la corporación}

Antaño, la Corporación diversificada podía simplemente puntualizar a sus unidades comerciales los mercados de productos terminales específicos y exhortarlas a convertirse en líderes mundiales. Pero con el cambio cada vez más rápido de los linderos del mer- cado, los objetivos son esquivos y su logro es a lo más, temporal. Unas pocas compañías han probado ser, ellas mismas, adeptas a inventar nuevos mercados y cambiar dramáticamente los patrones de escogencia de los clientes en mercados establecidos. Estas son las que hay que emular. La tarea crítica de la administración es crear una organización capaz de inspirar productos de funcionalidad irresistible o, aún mejor, crear productos que los clientes necesitan pero que todavía no han sido imaginados.

Esta es una tarea engañosamente difícil. En últimas, se requiere un cambio radical en la administración de grandes compañías. Esto significa, primero que todo, que las administraciones ejecutivas de las compañías occidentales deben asumir la responsabilidad de la decadencia competitiva. Todo el mundo conoce las altas tasas de interés, el proteccionismo japonés, las anticuadas leyes sobre antimonopolio, los estrepitosos sindicatos, y los inversionistas impacientes. Lo que es más difícil de ver, o más duro de aceptar, es el tan poco impulso adicional que las compañias en real idad reciben de la "ayuda" política o macroeconómica. Tanto la teoría como la práctica de la administración occidental han creado una traba en nuestro avance. Son los principios de la administración los que necesitan la reforma.

El enfrentar nuevamente a NEC y GTE es aleccionador y solamente analizamos uno de muchos de tales casos comparativos para comprender la base cambiante del liderazgo global. A principios de los 70, NEC articuló un intento estratégico para explo- 
tar la convergencia de computación y comunicaciones, lo que llamó "C\&C". El éxito, razonó la alta administración, dependería de las competencias adquiridas, particularmente en semiconductores. La administración adoptó una apropiada "arquitectura estratégica", resumida por el $\mathrm{C} \& \mathrm{C}$, y luego comunicó su intención a toda la organización y al mundo exterior hacia mediados de los 70 .

NEC constituyó un "Comité C\&C" de altos administrativos para inspeccionar el desarrollo de productos esenciales y competencias fundamentales. NEC puso en su sitio a grupos de coordinación y comités que pasaban a través de los intereses de negocios individuales. Consistente con su arquitectura estratégica, NEC trasladó enormes recursos para fortalecer su posición en componentes y procesadores centrales. Utilizando arreglos de mutua colaboración para multiplicar los recursos internos, NEC fue capaz de acumular una amplia colección de competencias fundamentales.

NEC identificó cuidadosámente tres corrientes interrelacionadas de evolución tecnológica y mercantil. La alta gerencia determinó que la computación evolucionaría desde los grandes computadores centrales al procesamiento distribuido, los componentes desde los sencillos IC al VLS1, y las comunicaciones desde el intercambio mecánico a través de la valla hasta los complejos sistemas digitales que ahora denominamos ISDN. En la medida en que las cosas evolucionaran más, pensó NEC, los negocios de computación, comunicaciones y componentes se trasladarían de tal manera que sería muy difícil distinguirlos, y habría enormes oportunidades para cualquier compañía que hubiera construido las competencias necesarias para servir todos los tres mercados.

La alta gerencia de NEC determinó que los semiconductores serían el "producto central" más importante de la compañía.

\section{¿Por qué entró NEC en miriadas de alianzas entre 1980 y 1988 ? \\ Para aprender y absorber las destrezas de ctras compañias.}

Entró en miríadas de alianzas estratégicas (más de 100 hasta 1987) con el propósito de construir competencias rápidamente y a bajo costo. En computadores centrales, su relación más famosa fue con Honeywell y Bull. Casi todos los arreglos mutuos en el campo de semiconductores y componentes fueron orientados hacia el acceso cae la tecnología. En la medida en que los gerentes operativos de NEC entraban en arreglos mutuos, comprendieron la razón de estas alianzas y el objetivo de internalizar las habilidades de los socios. El director de investigaciones de NEC resumió así la adquisición de competencia durante los 70 y los 80: "Desde el punto de vista de una inversión, era más rápido y más barato utilizar la tecnología foránea. No había necesidad de que nosotros desarrolláramos nuevas ideas".

Tal claridad de intento estratégico y de arquitectura estratégica no parecía 
existir en GTE. A pesar de que los ejeculivos más antiguos discutian las implicaciones de la evolucionante in- dustria de la tecnología de la información, no se dio a conocer ampliamente el punto comúnmente aceptado de cuáles compeiencias se requeririan para competir en esa industria. Mientras que el personal realizaba trabajo significaivo para identificar las tecnologias claves, los gerentes de línea de más rango, conıinuaban actuandocomo si estuvieran administrando unidades de negocios independientes. $\mathrm{La}$ descentralización dificultó enfocar las competencias esenciales. En lugar de eso, los negocios individuales se volvieron cada vez más dependientes de exiraños en cuanto a las trabilidades críticas y la colaboración se volvió una ruta para éxitos preparados. Hoy, con un nuevo equipo administrativo en el sitio, la GTE se ha reestablecido para aplicar sus competencias a mercados emergentes en servicios de velecomunicaciones.

\section{Las raíces de la ventaja competitiva}

La dilerencia que observamos sobre la manera en que NEC y GTE se conciberl a sí mísmas (un portalolio de competencias contra un portafolio de negocios) se repitió a través de muchas industrias. De 1980 a 1988 Canon creció en un $264 \%$, Honda en un 200\%. Compare eso con Xerox y Chrysler. $Y$ si los administradores occidentales estuvieron alguna vez inquietos por el bajo costo y la buena calidad de las imponaciones japoneses, ahora están apabullados por el ritmo con el que los rivales japoneses están inventando nuevos mercados, creando nuevos productos y mejorándolos. Canon nos ha dado copiadoras personales; Honda ha pasado de motocicletas a buggies de cuatro ruedas para moto-cross. Sony desarrolló el encordonador de leva, Yamaha, el piano digital. Komalsu desarrolló un bulldozer submarino de control renoto en tanto que el úlimo gambito de Cásio es un televisor LCD a color de pantalla pequeña ¿Quién hubiera ancicipado la evolución de estos mercados de vanguardia?

En mercados más eslablecidos, el reto japonés ha sido igualmente inquietante. Las compañías japonesas están generando un alud de características y mejoramientos funcionales que aplican la sofisticación tecnológica a los productos rutinarios. Los productores de carros japoneses han estado liderando la dirección en las cuatro ruedas, los motores de cilind ros de cuatro váivulas, sistemas de navegación incorporados en los carros y sofisticados sistemas de conirol electrónico de motores. Basado en la solidez de las características de sus productos, $\mathrm{Ca}$ non es ahora un compecidor en máquinas de transmisión de facsímiles, impresoras láser de escritorio y también de equipo de fabricación de semiconductores.

A corto plazo, la competitividad de una compañia se deriva de los alributos de precio/desempeño de los productos corrientes. Pero los sobrevivientes de la primera oleada de competición global, Larito occidentales como japoneses, todos estár convergiendo en formidables y similares estándares 
en cuanto a costo y cualidad del producio - las minimas barreras en la compelición conturuada, pero cada vex menos importante como fuentes de ventaja diferencial. A largo plazo, la competitividad se deriva de la habilidad para construir a menor costo y con mayor rapidez que los competidores, las competencias esenciales que producen arlículos no previstos. Las fuentes verdaderas de la ventaja se encuentran en la habilidad de la administración para consolidar en competencias a lo largo y ancho de la corporación que den poder a los negocios individuales para adaplarse räpidamente a las cambiantes oportunidades.

Los ejecutivos más antiguos que claman no poder construir competencias porque sienter que la autonomía del negocio es sacrosanta o porque sus manos están atadas a la lumbre del presupuesto trimestral, deberian pensarlo de nuevo. El problema en muchas compañias occidentales no es que sus ejecutivos más antiguos sean menos capaces que los del Japón 0 que las compañías japonesas posean mayores capacidades lécnicas. Más bien, es su apego a un concepto de Corporación que necesariamente limila la habilidad de los negocios individuales para explotar lotalmente el hondo depósito de capacidad tecnológica que poseen muchas compañias curopeas y americanas.

La corporación diversificada es como un árbol frondoso, E tronco y las ramas más grandes son los productos centrales, las ramas más pequeñas son las unidades comerciales; las hojas, las flores y los frutos son los productos terminales. El sıtema de raíces que, provee alimento, sustento y estabilidad es la competencia esencial. Usted puede pasar por alto la solidez de los competidores mirando sólo los productos terminales, de la misma manera que usted puede pasar por alto la fortaleza de un árbol si sólo mira sus hojas (Vercuadro "Compelencias: Las Aaices de la compelilinldad", pág. 72).

Las compelencias esenciales son el aprendizaje colectivo en la organización, especialmente cómo coordinar diversas habilidades de producción e integrar múltiples corrientes de tecnologías. Considere la capacidad de SONY para miniaturizar o la maestría de PHJL.IPS para los medios ópticos. El conocimiento teórico para poner un radio en una ficha no le asegura por sî mismo a una compañía la destreza para producir un radio miniatura no más grande que una larjeta de presentación. Para realizar esta proeza, Casio debe armonizar el conocimiento en minialuriaación con el diseño de microprocesadores, la ciencia pertinente al caso y la caja de precisión ultradelgada (las mismas destrezas que aplica a sus calculadoras miniatura, a los televisores de bolsillo y a los relojes digilales .

\section{A diferencia de los activos fisicos, las competencias no se deterioran cuando se aplican o se comparten. Ellas crecen.}

Si la competencia central es sobre cómo armonizar corrientes de tecnologia, es también sobre la organiza- 
Competencias: Las Raices de la Competitividad

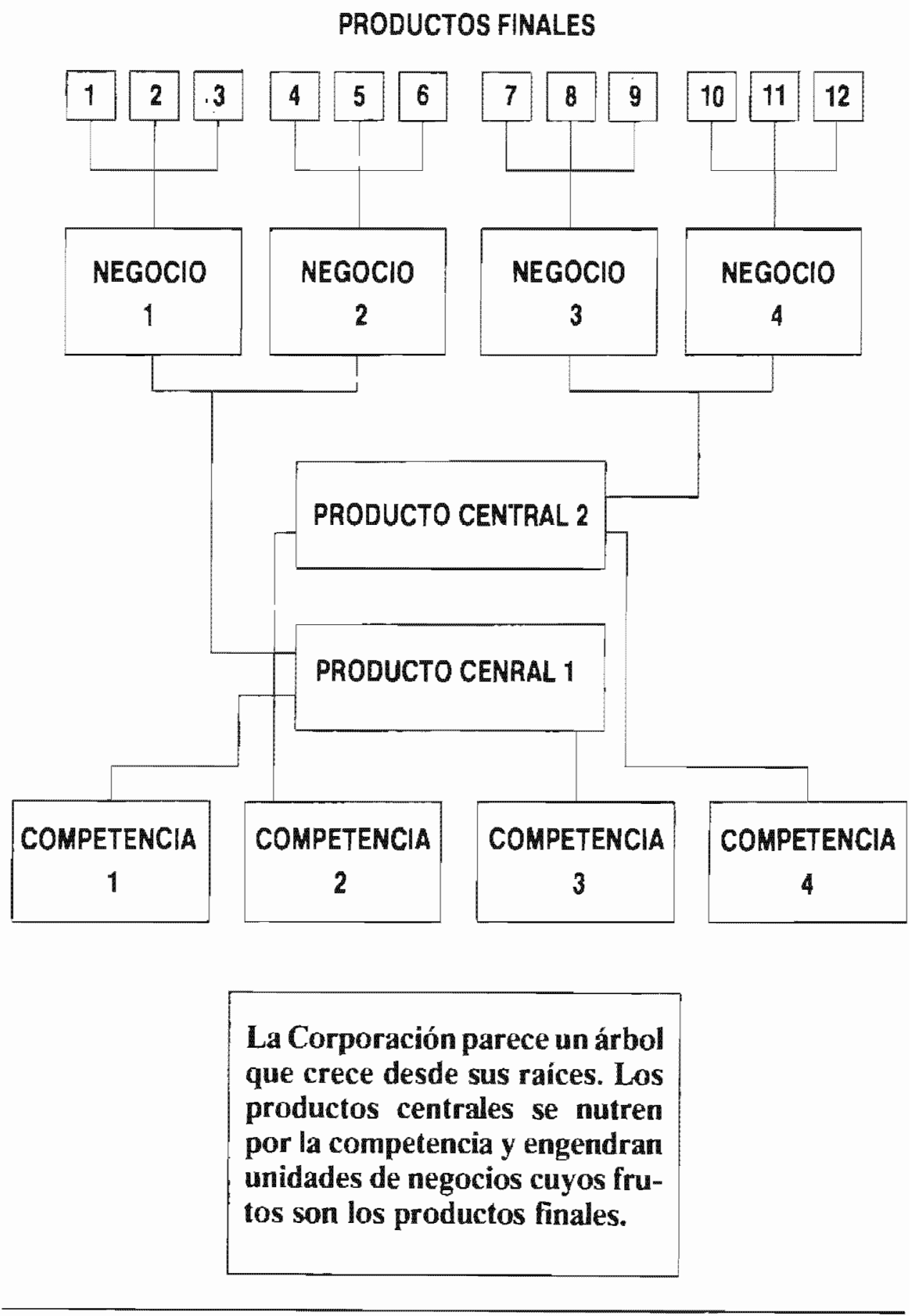


ción del trabajo y la entrega del valor.

Entre las competencias de SONY está la miniaturización. Para lievar la miniaturización a sus productos, SONY debe asegurar que los tecnólogos, los ingenieros y los comerciantes tengan una comprensión compartida de las necesidades del cliente y de las posibilidades tecnológicas. La fuerza de la competencia central se siente decisivamente en los servicios y en la manufactura. Citicorp estuvo adelante de otros por invertir en un sistema operativo que le permitió participar en el mercado mundial 24 horas al día. Su competencia en sistemas ha dado a la compañía los medios para diferenciarse de muchas instituciones de servicios financieros.

La competencia central es comunicacación, entrega y un profundo compromiso de trabajar a través de límites organizacionales. Esto envuelve muchos niveles de personas y todas las furciones. La investigación de tipo mundial, por ejemplo, en láseres o cerámica, puede tener lugar en laboratorios corporativos sin tener un impacto en cualquiera de los negocios de la compañía. Las destrezas que juntas constituyen la competencia central, deben unirse alrededor de individuos cuyos esfuerzos no se enfoquen en forma tan estrecha que no puedan reconocer las oportunidades para mezclar su maestría funcional con las de otros, en nuevas e interesantes maneras.

La competencia central no disminuye con el uso. Al contrario de los activos físicos, que sí se deterioran con el tiempo, las competencias se acrecien- tan a medida que se aplican y comparten. Pero aun las competencias necesitan ser alimentadas y protegidas; el conocimiento se desvanece si no se usa. Las competencias son el pegante que une los negocios existentes. También son el motor de desarrollo de nuevos negocios. Los patrones de diversificación y acceso al mercado pueden ser guiados por las competencias, no sólo por la atracción de los mercados.

Considere la competencia de $3 \mathrm{M}$ con la cinta pegante. Al soñar con negocios tan diversos como las notas "Póngala al correo", la cinta magnética, el film fotográfico, cintas sensitivas a la presión y abrasivos revestidos, la compañia ha llegado a soportar competencias ampliamente compartidas en sustratos, revestimientos y adhesivos y ba ideado varias maneras de combinarlas. Ciertamente, $3 \mathrm{M}$ ha invertido en ellas consistentemente. Lo que parece ser un portafolio extremadamente diversificado de negocios, disfraza unas cuantas competencias centrales compartidas.

En contraste, bay grandes compañías que han tenido el potencial para construir competencias centrales, pero fallaron en hacerlo porque la alta gerencia fue incapaz de concebir la compañía como otra cosa que no fucra una colección de negocios separados. GE vendió mucho de su negocio de electrónica hogareña a la Thomson de Francia, alegando que se estaba volviendo cada vez más difícil mantener la competitividaa en ese sector. Sin lugar a dudas eso fue asi, pero es irónico que vendiera varios negocios 
claves a competidores que ya eran líderes en competencia -Black \& Decker - en pequeños motores eléctricos y, Thomson, que estaba ansiosa por construir su competencia en microelecurónica y había aprendido de los japoneses que una posición en electrónica consumista era vilal para este reto.

La administración, atrapada en la decisión de la unidad estratégica de negocios (UEN) casi inevitablemente encuentra sus negocios individuales dependientes de fuerzas externas en lo que se refiere a componentes críticos, tales como motores o compresores. Pero éstos no son sólo componentes. Son productos esenciales que contribuyen a la competitividad de una ampiia variedad de productos terminales. Son las personificaciones fisicas de las competencias centrales.

\section{Cómo no pensar en competencia}

Desde que las compañias están en una carrera para construir competencias que deierminen el liderazgo global, las compañías exitosas han cesado de imaginarse a sí mismas como manojos de negocios que fabrican productos. Canon, Honda, Casio o NEC pueden parecer que presiden los portafolios de negocios no relacionados en términos de consumidores, canales de distribución y estrategia de comercialización. Ciertamente, ellas tienen portafolios que pueden parecer idiosincráticos a veces: NEC es la única compañía global que está entre los líderes en computación, telecomunicaciones y semiconductores y que tiene un flore- ciente negocio de electrónica consumista.

Pero las apariencias engañan. En NEC, ia tecnología digital, especialmente el VLSI y las habilidades de integración de sistemas, es fundamental. En las competencias centrales que las fundamenta, negocios discordes se hacen coherentes. Es la competencia esencial de Honda en motores y trenes eléctricos lo que le da una clara ventaja en negocios de carros, motocicletas, cortadoras de césped y generadores.

Las competencias centrales de Canon en óptica, copias y controles de microprocesador la ban capacitado para entrar y aun dominar mercados aparentemente tan diversos como copiadoras, impresoras a láser, cámaras y buscadores de imagen. Philips trabajó por más de 15 años para perfeccionar su competencia en medios ópticos (disco láser), como lo hizo JVC en consiruir una posición de liderazgo en video grabación. Otros ejemplos de competencias centrales podrían incluir la mecanotrónica (la habilidad para unir la ingeniería electrónica con la ingeniería mecánica) exhibiciones de video, bioingeniería y microelectrónica. En las primeras etapas de la construcción de su competencia, Philips no pudo haber imaginado todos los productas que resultarían de su competencia en medios opticos ni JVC pudo haber anticipado los encordadores miniatura cuando por primera vez empezó a explorar las tecnologías de video-cintas. A diferencia de la batalla por el dominio global de marcas, que es visible en los medios de prensa y radiodilusión del mundo, y 
que apunta a la construcción de "compartir la mente" en forma global, la lucha para construir competencias de clase mundial es invisible para la gente que deliberadamente no la está buscando. La alta gerencia a menudo les sigue la pista al costo y a la calidad de los productos de los competidores, ¿pero cuántos administradores desenredan la telaraña de alianzas que sus competidores japoneses ban construido para adquirir competencias a bajo costo? ¿En cuántos salones de juntas occidentales hay una comprensión explícita y compartida de las competencias que la compañía debe construir para el liderazgo mundial? En verdad, ¿cuánios ejecutivos de más alto rango discuten la distinción crucial entre la estrategia competitiva a nivel de un negocio y la estrategia competitiva a nivel de toda una compañía?

Seamos claros. Cultivar la competencia central no significa gastar más que los rivales en investigación y desarrollo. En 1983, cuando Canon sobrepasó a Xerox en compartir el mercado unitario a nivel mundial, del negocio de copiadoras, su presupuesto R\&D en reprografía fue sólo una fracción del de Xerox. Durante los últimos 20 años, NEC ha gastado menos en $R \& D$ como un porcentaje de ventas que casi todos sus competidores americanos y europeos.

La competencia central tampoco significa costos compartidos, como cuando dos o más SBU usan una facilidad común -una fábrica, facilidad de servicio o fuerza de ventas- o comparten un componente común. Las ganancias del compartir pueden ser sustanciales, pero la búsqueda de costos compartidos es típicamente un esfuerzo "post hoc" (después del hecho) para racionalizar la producción a través de negocios existentes, no un esfuerzo premeditado para construir las competencias de las cuales crecen por los mismos negocios.

Además, el construir las competencias centrales es más ambicioso y diferente que integrarse verticalmente. Los gerentes que deciden si bacer o comprar, empezarán con productos terminales y miran corriente arriba las eficiencias de la cadena de suministros y corriente abajo la distribución y los clientes. Ellos no hacen inventario de habilidades y esperan aplicar esas habilidades de maneras no tradicionales. (Por supuesto, las decisiones sobre las competencias sí proveen una lógica para la integración vertical. Canon no está par. ticularmente integrada en su negocio de copiadoras, excepto en aquellos aspectos de la cadena vertical que apoya las competencias que considera críticas).

\section{Identificación de competencias esenciales y su pérdida}

Por lo menos tres pruebas pueden aplicarse para identificar las competencias esenciaies en una compañía.

Primero, una competencia esencial provee acceso potencial a una amplia variedad de mercados. La competencia en sistemas de exhibición, por ejemplo, capacita a una compañía para participar en negocios tan diversos como calculadoras, aparatos miniatura de televisión, monitores para 
computadoras portátiles y tableros de instrumentes para automóviles - lo que explica que la entrada de Casio en el mercado de televisores de mano era predecible. Segundo, una competencia esencial deberia hacer una contribución significativa a los beneficics que el cliente percibe del producto final. Claramente, el conocimiento especializado sobre motores de la Honda llena este programa.

Finalmente, una competencia esencial debe ser dificil de imitar por los competidores. Y será dificil si es una armonización compleja de tecnologias individuales y habilidades de producción. Un rival podria adquirir algunas de las tecnologias que comprenden la competencia central, pero encontrará que es más dificil duplicar el patrón más o menos comprensivo de la coordinación y aprendizaje internos. La decisión de JVC a comienzos de los 60 de continuar el desarrollo de una competencia sobre videocintas, pasó las tres prucbas delineadas. La decisión de RCA a finales de los 70 para desarrollar un sistema de video casetera basado en estilete no pasó las pruebas.

Pocas compañias están dispuestas a construir un liderazgo mundial en más de cinco o seis competencias fundamentales. Una compañia que compile una lista de 20 a 30 aptitudes probablemente no ha producido una lista de competencias esenciales. Sin embargo, es probablemente buena disciplina generar una lista de este tipo y ver agregar capacidades como bloques de construcción. Esto tiende a impulsar la búsqueda de tratos autorizados y alianzas a través de las cuales la com- pañia puede adquirir las pièas faltantes, a bajo costo.

La mayoría de las compañias occidentales dificilmente piensan sobre la competitividad en estos términos. Es bora de echar una concienzuda mirada a los riesgos que corren. Las compañias que juzgan la competitividad propia y la de sus competidores, principalmente en términos de precio/desempeño de los productos terminales, están cortejando la erosión de las competencias esenciales - o haciendo muy poco esfuerzo para relevirlas. Las destrezas enclavadas que dan lugar a la siguiente generación de productos competitivos no pueden ser "alquiladas" localizando fuentes de abastecimiento o por el tipo de relaciones OEM de suministros. Desde nuestro punto de vista, demasiadas compañias han rendido inconscientemente competencias fundamentales cuando redujeron la inversión interna en lo que erróneamente pensaron que eran sólo "centros de costos" a favor de proveedores externos.

\section{A diferencia de Chrysler, Honda nunca cederia la responsabilidad de la manufactura de sus motores y mucho menos el diseño de ellos.}

Considere a Chrysler. A diferencia de Honda, se ha inclinado a considerar los motores y los trenes eléctricos sencillamente como un componente más. Chrysler se está volviendo, cada vez más, dependiente de Mitsubishi y de Hyundai: entre 1985 y 1987 , el número de motores modificados subió de 
252.000 a $38 z .000$. Es difícil imaginar a Honda cediendo responsabilidad y mucho menos el diseño de una parte tan crítica del funcionamiento de un carro a una compañía extraña -que es por lo cual Honda ha hecho tan enorme compromiso con los autos de carreras Fórmula Uno. Honda ba sido capaz de agrupar sus tecnologías relacionadas con motores; conferenciando, las ha introducido en una competencia enorme de la cual desarrolla productos de impacto mundial, a pesar de que los presupuestos $R \& D$ son menores que los de GM y Toyota. Por supuesto, es perfectamente posible que una compañía tenga un producto competitivo ísto, pero estar rezagaca en el desarrollo de competencias fundamentales --al menos por algún tiempo. Si una compañía quisiera entrar hoy al negocio de copiadoras, encontraría una docena de compañías japonesas más gustosas de suministrar las copiadoras sobre la base de una etiqueta privada OEM. Pero cuando las tecnologías fundamentales cambian o si el proveedor decide entrar personalmente en el mercado y convertirse en un competidor, la línea de productos de esa compañía, podría ser vulnerable junto con todas sus inversiones en mercadeo y distribución. Tomar ventaja en la fuente, puede proveer un atajo hacia un producto más competitivo, pero ésto típicamente contribuye poco a construir las destrezas incorporadas en la gente que son necesarias para sostener el liderazgo del producto.

Tampoco es posible que una compañía tenga una alianza inteligente o una estrategia original si no ha hecho una selección de dónde construirá el liderazgo de competencia. Claramente, las compañías japonesas se han beneficiado de las alianzas. Las han utilizado para aprender de los socios occidentales quienes no estaban totalmente comprometidos a preservar sus propias competencias esenciales. Como lo hemos argüido anteriormente en estas páginas, el aprendizaje dentro de una alianza requiere un compromiso positivo de recursos - viajes, agrupamiento de personas dedicadas, facilidades apoyadas en pruebas, tiempo para internalizar y verificar lo que se ha aprendido. Puede que una compañía no haga el esfuerzo si no tiene metas claras sobre construcción de competencias.

Otra manera de perder es renunciar a las oportunidades en favor de competencias establecidas que están evolucionando en negocios existentes. En los 70 y los 80 , muchas compañías americanas y europeas - como GE, Motorola, GTE, Thorn, GEC - escogieron retirarse del negocio de televisión a color, que consideraron maduro. Si por "maduro" querían significar que habían agotado ideas de productos nuevos precisamente en el momento en que los rivales globales habían enfocado el negocio de TV para entrar, entonces sí, la industria estaba madura. Pero ciertamente no estaba madura en el sentido de que estaban agotadas todas las oportunidades para realizar y aplicar las competencias basadas en video.

Al deshacerse de los negocios de televisión, estas compañías fallaroñ en distinguir entre despojarse del negocio y destruir sus competencias de video 
basadas en los medios de comunicación. No solamente salieron del negocio de TV sino que cerraron la puerta ra toda una corriente de futuras oportunidades que se apoyan en competencias basadas en video. La industria de lá televisión, considerada inatractiva por muchas compañias de Estados Unidos en los 70, es hoy el centro de un fiero debate político público sobre la inhabilidad de las corporaciones de U.S. para beneficiarse de una oportunidad de $\$ 20$ billones al año que será lo que le representará a HDTV hacia mediados y postrimerías de los 90. Irónicamente se le ha pedido al gobierno de U.S. que financie un proyecto masivo de investigación - de hecho, que compense a las compañías norteamericanas por su fracaso en preservar las competencias fundamentales críticas cuando tuvieron la oportunidad.

En contraste, uno puede ver una compañía como Sony reducir su énfasis en VCRs (en los que no ha tenido mucho éxito y en donde ahora amenazan las compañías coreanas) sin reducir su compromiso con las competencias relacionadas con video. El betamax de Sony condujo a un desastre. Pero emergió consevando intactas sus competencias de grabación de videocintas y actualmente está retando a Matsushita en el mercado de encordonador de $8 \mathrm{~mm}$.

Hay aquí dos claras lecciones. Primera, los costos de perder una competencia fundamental se pueden calcular por anticipado sólo parcialmente. El bebé puede ser arrojado con el agua de la bañera en decisiones de abandonar. Segunda, puesto que las compe- tencias fundamentales se construyen a través de un proceso de continuo mejoramiento y realce que puede abarcar una década o más, una compañía que no ha invertido en la construcción de competencias esenciales encontrará muy difícil entrar a un mercado emergente, a menos que, por supuesto, esté contenta de servir simplemente de canal de distribución.

Compañías americanas de semiconductores como Motorola aprendieron esta dolorosa lección cuando eligieron renunciar a la participación directa en la generación $256 \mathrm{~K}$ de fichas DRAM. Habiendo pasado por alto este asalto, Motorola, como la mayoría de los competidores americanos, necesitaba una gran infusión de ayuda técnica de los socios japoneses para sumarse a la batalla por la generación del mega byte $1^{*}$. Cuando se trata de competencias fundamentales, es difícil bajarse del tren, caminar hasta la siguiente estación y volver a abordarlo.

\section{De las competencias esenciales a los productos centrales}

El vínculo tangible entre competencias esenciales y productos terminales, es lo que llamamos productos centrales - la incorporación física de una o más competencias esenciales. Los motores de Honda, por ejemplo, son productos centrales, pasadores entre habilidades de diseño y desarrollo que en últimas conducen a una proliferación de productos terminales. Los productos centrales son los componentes o sub-ensamblajes que realmente con-

-Byte = Agrupación de 8 bits (N. del T.) 
tribuyen al valor de los productos terminales. Pensar en términos de productos centrales obliga a una compañía a distinguir entre la participación en marcas que logra en los mercados de productos terminales (por ejemplo, $40 \%$ del mercado de neveras de los Estados Unidos) y la participación en manufacturas que logra de cualquier producto central particular (por ejemplo, $5 \%$ de la participación mundial de la producción totai de compresores).

Canon es famosa por tener un $84 \%$ de la participación en la manufactura mundial de "motores" para impresoras láser portátiles, aunque su participación de marca en el negocio de impresoras láser es minúscula. De igual manera, Matsushita tiene una participación en la manufactura mundial del $45 \%$ en componentes claves VCR, muchísimo más de su participación de marca (Panasonic, JVC, y otros) que es del 20\%. Y Matsushita tiene una participación dominante de productos centrales en compresores a nivel mundial, estimada en $40 \%$, aunque es pequeña su participación de marca tanto en negocios de aire acondicionado como de neveras.

\section{Mantenga el dominio de la manufactura mundial de productos centrales, y así conservará el poder de dar forma a la evolución de productos terminales.}

Es esencial hacer esta diferenciación entre competencias fundamentales, productos centrales y productos ter- minales, porque la competición global se juega con diferentes reglas y con diferentes intereses en cada nivel. Al construir o defender el liderazgo a largo plazo, una compañía saldrá ganadora en cada nivel. A nivel de competencia esencial la meta es construir el liderazgo mundial en el diseño y desar rollo de una clase particular de funcionalidad del producto, sea ésta el almacenamiento y recuperación de datos en forma compacta, como la competencia de medios ópticos de Philips, o ia compactibilidad y facilidad de uso, como ocurre con los controles de micromotores y microprocesadores de Sony.

Para sostener el liderazgo en las áreas escogidas de competencias esenciales, estas compañias buscan llevar al máximo su participación en la manufactura mundial de productos centrales. La manufactura de productos centrales para una amplia variedad de clientes externos (e internos) produce la renta y los resultados del mercado que, por lo menos parcialmente, determina el ritmo al que las competencias esenciales pueden ser mejoradas y proyectadas. Esta manera de pensar estaba respaldando la decisión de JVC hacia mediados de los 70 de establecer relaciones de suministro de VCR con compañías que son líderes nacionales en Europa y en los Estados Unidos. Al proveer a Thomson, Thorn y Telefunken (todas compañías independientes en ese tiempo, así como a los socios estadounidenses, JVC fue capaz de ganar el efectivo y la diversidad de la experiencia mercantil que últimamente la capacitó para sobrepasar a Philips y a Sony). Philips de- 
sarrolló competencias en video-cintas paralelamente con JVC pero falló al construir una red mundial de relaciones OEM que le habrían permitido acelerar el refinanciamiento de su competencia en video-cintas a través de la venta de productos esenciales.

El éxito de JVC no se ha perdido en compañías coreanas como Goldstar, Samsung, Kia y Daewoo, que están construyendo liderazgo en productos esenciales en áreas tan diversas como exhibidores, semiconductores y motores para automóviles a través de sus contratos de suministros OEM con compañías occidentales.

Su objetivo reconocido es quitarles la iniciativa de inversiones a los competidores potenciales, con frecuencia compañías estadounidenses. Al hacerlo, aceleran los esfuerzos de construcción de competencias, mientras "ponen fuera" a sus competidores. Centrándose en competencia e incorporándolo a los productos esenciales, los competidores asiáticos han vigorizado primero los mercados de repuestos y luego han permitido que sus superiores productos se muevan corriente abajo para conseguir participación de marca. Y no es probable que permanezcan para siempre como proveedores baratos. A medida que se consolida su reputación en el liderazgo de marca, pueden ganar el liderazgo en precio. Honda ha probado esto con su línea ACURA y otros japoneses fabricantes de carros están haciendo lo mismo.

El control de los productos esenciales es crítico por otras razones. Una posi- ción dominante en productos esenciales le permite a una compañía dar forma a la evolución de aplicaciones y mercados terminales. Productos esenciales compactos de audio relacionados con discos, tales como conductores de datos y láseres, han permitido a Sony y a Philips influir en la evolución del negocio de computadores periféricos en el almacenamiento de medios ópticos. A medida que una compañía multiplica el número de áreas de aplicación para sus productos centrales, puede reducir consistentemente el costo, el tiempo y arriesgarse en el desarrollo de nuevos productos. En resumen, productos esenciales bien enfocados pueden conducir a economías de escala y extensión.

\section{La tiranía de SBC}

No se pueden comprender los nuevos términos del compromiso competitivo usando heramientas analíticas diseñadas para administrar la corporación diversificada de hace 20 años, cuando la competición era principalmente doméstica (GE contra Westinghouse, General Motors contra Ford) y todos los competidores claves hablaban el lenguaje de las mismas escuelas de negocios y consultorías. Las viejas recetas tienen efectos secundarios potencialmente tóxicos.

La necesidad de nuevos principios es más obvia en compañías organizadas exclusivamente de acuerdo con la lógica de SBUs. Las implicaciones de los dos conceptos alternos de la corporación están resumidos en "Dos Conceptos de la Corporación: SBU o Competencia Esencial". 
Dos conceptos de la corporación: SBU o competencia esencial

SBU

Bases de la Competitividad de los productos Competición de hoy.

Estructura Portafolio de negocios relacioCorporativa nados en término de productomercado.

Estatus de la La autonomia es sacrosanta; el Unidad

Comercial

Distribución

de los

Recursos

Valor

agregado

de la Alta

Gerencia
$S B U$ "posee" todos los recursos distintos al efectivo.

Negocios separados son la unidad de análisis; el capital se distribuye negocio a negocio.

Optimizar las utilldades corporativas a través de Intercambios de distribución de capifal entre los negocios.
COMPETENCIA ESENCIAL

Competición Interfirmas para construir competencias.

Portafolio de competencias, productos centrales y negocios.

SBU es un depósito potencial de competencias esenciales.

Los negocios y las corripetencias son la unidad de análisis: la alta gerencia distribuye el capital y el talento.

Enunciar competencias estratégicas de arquitectura y construcción para asegurar el futuro.
Obviamente, las corporaciones diversificadas tienen un portafolio de productos y un portafolio de negocios. Pero creemos en una concepción de la compañía también como un portafolio de competencias. Las compañías de U.S. no carecen de los recursos técnicos para construir competencias, pero la alta gerencia con frecuencia no tiene la visión para construirlas ni los medios administrativos para juntar los recursos esparcidos a través de múltiples negocios. Un cambio en el compromiso inevitablemente influirá en los patrones de diversificación, en el despliegue de habilidades, en las prioridades de distribución de los recursos y en los planteamientos de alianzas y localización de fuentes de abastecimiento.

Hemos descrito los tres diferentes planos en los que se libran las batallas por el liderazgo global: competencia fundamental, productos esenciales y pro- ductos finales. Una corporación tuene que saber si está ganando o perdiendo en cada plano. Por el solo peso de la inversión una compañía podría ser capaz de derrotar a sus rivales en tecnologías ilusorias y aun perder la carrera por la construcción del liderazgo en competencias esenciales. $\mathrm{Si}$ una compañía está ganando la carrera en construir competencias esenciales (contrario a construir liderazgo en unas pocas tecnologías), ciertamente sobrepasará a sus rivales en el desarrollo de nuevos negocios. Si una compañía está ganando la carrera por capturar participación en la manufactura mundial de productos esenciales, probablemente superará a los rivales en el perfeccionamiento de las características del producto y la proporción precio/desempeño.

Determinar si uno está ganando o perdiendo las batallas por productos finales es más difícil, porque las dispo- 
siciones sobre participación en el mercado no reflejan necesariamente la competitividad subyacente de varias compañias. Ciertamente, las compañias que tratan de cimentar la participación en el mercado confiando eo la competitividad de otras, en lugar de invertir en competencias fundamentales y en el liderazgo mundial de productos esenciales, pueden estar caminando en arena movediza. En la carrera por el dominio global de marca, compañías como 3M, Black \& Decker, Canon, Honda, Nec y Citicorp han construido sombrillas globales de marca haciendo que los productos proliferen fuera de las competencias fundamentales. Esto ha permitido que los negocios individuales creen imagen, lealtad de los clientes y acceso a los canales de distribución.

Cuando piense en esta reconceptualización de la corporación, la primacía del SBU - un dogma organizacional para una generación- es ahora claramente un anacronismo. Donde el SBU es un artículo de fe, la reşistencia a las seducciones de la descentralización pueden parecer heréticas. En muchas compañilas, el prisma SBU significa que sólo un plano de la batalla competitiva global, la batalla para poner hoy los productos competitivos en el estante, es visible a la alta gerencia. ¿Cuáles son las costos de esta distorsión?

Inversión inadecuada en el Desarrollo de Competencias Fundamentales $y$ Productos Esenciales. Cuando la organización se concibe como una multiplicidad, ningún negocio individual puede sentirse responsable por man- tener una visible posición en produc tos esenciales ni ser capaz de justificar la inversión requerida para cimentar el liderazgo mundial en alguna competencia fundamental. En ausencia de una opinión más comprensiva, impuesta por la administración corporativa, los gerentes de SBU tenderán a sub-invertir. Recientemente, companías como Kodak y Philips han reconocido esto como un problema potencial y ban comenzado a buscar nuevas formas organizacionales que les permitan desarrollar y fabricar productos esenciales para clientes intemos $y$ externos.

Los gerentes de SBU han concebido tradicionalmente a los competidores de la misma manera en que se ven a sí mismos. En general, han fallado en notar el énfasis que los competidores asiáticos estaban poniendo en la construcción de liderazgo en productor esenciales o en comprender el crítico vioculo entre el liderazgo en manufactura mundial y la habilidad para sostener el ritmo de desarrollo de competencias fundamentales. Fallaron en perseguir las oportunidades de suministro OEM o en mirar a través de las divisiones de productos varios en un intento de identificar las oportunidades para iniciativas coordinadas.

RECURSOS APRISIONADOS. A medida que evoluciona un SBU, frecuentemente desarrolla competencias únicas. Típicamente, la gente que encarna esta compelencia es considerada como la única propiedad del negocio en el que creció. El gerente de otra SBU que pide en préstamo gente con trlento, se expone a recibir un frío 


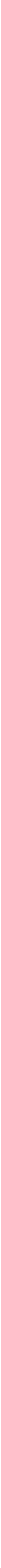


rechazo. Los gerentes SBU no sólo no desean piestar a sus portadores de competencia sino que de hecho pue"den esconcer el talento para prevenir su redesoliegue en la búsqueda de nuevas oportunidades. Esto puede compararse con los habitantes de un país subdesarrollado que esconden la mayor parte del dinero en efectivo debajo de los colchones. Los beneficios de las competencias, como los beneficios del suministro de dinero, dependen de la velocidad de la circulación así como de la cantidad de mercancías almacenadas de que disponga.

Las compañias occidentales han tenido tradicionalmente una ventaja en la ex stencia de habilidades que poseen. ¿Pero han sido capaces de redelinearlas rápidamente para responder a nuevas oportunidades? Canon, NEC y Honda han tenido una menor existencia de la gente y las tecnologías que componen las competencias fundamentales, pero pudieron moverla mucho más rápido de una unidad de negocios a otra. Que el R\&D corporativo gaste en Canon no es plenamente indicativo del tamaño de la existencia de competencias fundamentales de Canon y no le dice nada al observador casual sobre la velocidad con que Canon es capaz de mover las competencias fundamentales para sacar partido de las oportunidades.

\section{Es extraño que los gerentes SBU deban estar hechos para competir por el efectivo corporativo pero nunca por la gente clave.}

Cuando las competencias se aprisionan, la gente que las lleva no es asignada a las oportunidades más excitantes y sus habilidades empiezan a atrofiarse. Solamente liberando totalmente las competencias esenciales, pueden las pequeñas compañías como Canon darse el lujo de competir con gigantes de la industria como Xerox. Es extraño que los gerentes SBU que están perfectamente dispuestos a competir por el efectivo en el importante proceso de presupuestar, no están dispuestos a competir por la gente -el más precioso activo de la compañía. Encontramos irónico que la alta gerencia dedique tanta atención al importante proceso de presupuestar. Los administradores de más alto rango rara vez son capaces de mirar hacia cuatro o cinco niveles inferiores en la organización, identificar la gente que encarna competencias críticas y moverla a través de los linderos organizacionales.

INNOVACION LIMITADA. Si no se reconocen las competencias esenciales, los SBUs individuales perseguirán solamente aquellas oportunidades de innovación que están a la mano - extensiones marginales de líneas de productos o expansiones geográficas. Las opor tunidades híbridas como máquinas Fax, computadores de tipo maletín, televisores portátiles o teclados musicales portátiles, sólo emergerán cuando los gerentes se quiten sus gafas SBU. Recuerde, Canon apareció en el negocio de cámaras fotográficas cuando se estaba preparando para convertirse en líder mundial de copiadoras. Concebir la corporación en términos de competencias fundamen- 
tales amplia el dominio de la innovación.

\section{Desarrollo de arquitectura estratégica}

La fragmentación de las competencias fundamentales se torna inevitable cuando los sistemas de información de una compañía diversificada, los patrones de comunicación, las trayectorias profesionales, las recompensas administrativas y los procesos de la estrategia de desarrollo, no trascienden las líneas de SBU. Creemos que la administración de más alto rango debería gastar una significativa cantidad de tiempo en desarrollar una arquitectura estratégica a lo largo y ancho de la corporación, que establezca objetivos para cimentar la competencia. Una arquitectura estratégica es un mapa vial del futuro que identifica las competencias fundamentales que hay que cimentar y sus tecnologias constituyentes.

Proveyendo un impulso al hecho de aprender de las alianzas y un foco a los esfuerzos internos de desarrollo, una arquitectura estratégica con la $\mathrm{C} \& \mathrm{C}$ de NEC puede reducir dramaticamente la inversión que se necesita para asegurar el liderazgo futuro del mercado. ¿Cómo puede una compañía hacer sociedades inteligentemente sin una clara comprensión de las competencias fundamentales que está tratando de cimentar y las que está intentando prevenir de ser transferidas sin intención?

Por supuesto, todo esto hace preguntar qué formas deberia tener una arquitectura estratégica. La respuesta será diferente para cada compañía. Pero es útil pensar otra vez en ese árboi, en la corporación organizada alrededor de productos finales $y$, en definitiva, en competencias fundamentales. Para echar raices suficientemente fuertes, una compañia debe responder algunas preguntas fundamentales:

¿Cuánto tiempo podríamos preservar nuestra competitividad en este negocio si no controlamos esta particular competencia esencial? ¿Qué tan importante es esta competencia esencial para los beneficios percibidos del cliente? ¿Qué oportunidades futuras serian excluidas si perdiéramos esta competencia particular? Además, la arquitectura provee una lógica a la diversificación del producto y del mercado.

Se le preguntaria a un gerente SBU: ila nueva oportunidad de mercado agrega algo a la meta general de convertirse en el mejor competidor del mundo?

¿Saca partido de o añade algo a la competencia fundamentai? En Vickers, por ejemplo, las opciones de diversificación han sido juzgadas en el contexto de convertirse en la mejor compañía del mundo en el control del movimiento y la energía (ver el inserto "Vickers Aprende el Valor de la Arquitectura Estratégica").

La arquitectura estratégica debe establecer prioridades en la distribución de los recursos que sean transparentes para toda la organización. Esto le proporciona a la alta gerencia un modelo para las decisiones de distribución de los recursos. Ayuda a los gerentes de bajo nivel a comprender la lógica de las prioridades de la asig- 
nación y disciplina a los gerentes de más rango para mantener la consistencia. En resumen, produce una definición de la compañía y los mercados que sirve. $3 \mathrm{M}$, Vickers, NEC, Canon y Honda, todos califican en este respecto. Honda sabia que estaba sacando provecho de lo que había aprendido de las motocicletas - cómo lograr altas revoluciones, marcha uniforme, motores livianos- cuando entró al negocio de carros. La tarea de crear una arquitectura estratégica obliga a la organización a identificar y comprometerse con los vínculos técnicos y de producción a través de SBUs, lo que dará una clara ventaja competitiva.

Es la consistencia en la asignación de recursos y el desarrollo de una apropiada infraestructura administrativa, lo que infunde vida a una arquitectura estratégica y crea una cultura administrativa, equipos de trabajo, una capacidad para cambiar y una voluntad para compartir los recursos, para proteger la propiedad de las habilidades y para pensar a largo plazo. Esta es también la razón por la cual la arquitectura específica no puede ser copiada fácilmente o de la noche a la mañana por los competidores. La arquitectura estratégica es una herramienta para la comunicación con los clientes y otros constituyentes externos. Revela la dirección general sin descubrir cada paso.

\section{Redespliegue para sacar partido de las competencias}

Si las competencias fundamentales de la compañía son su recurso crítico y si la alta gerencia debe asegurar que los portadores de la competencia no sean retenidos como rehenes por algún negocio particular, entonces se deduce que los SBUs deben licitar por las competencias esenciales de la misma manera como licitan por los recursos. Hemos establecido este punto de refilón. Es lo suficientemente importante como para considerarlo más detenidamente.

Una vez que la alta gerencia (con la ayuda de gerentes de división y de SBU) ha identificado las competencias abovedadas, debe solicitar a los negocios que identifiquen los proyectos y la gente estrechamente relacionada con ellos.

Los empleados corporativos deben dirigir una auditoría de la ubicación, número y calidad de la gente que encarna la competencia.

Esto envía una señal importante a los mandos medios: las competencias fundamentales son recursos corporativos y deben ser redistribuidos por la administración corporativa. Un negocio individual no posee a nadie. Los SBU tienen derecho a los servicios de empleados individuales en tanto que la administración SBU pueda demostrar que la oportunidad que persigue da el más alto beneficio posible de invertir en sus habilidades. Este mensaje se acentúa más si cada año, en la planeación estratégica o en el proceso presupuestal, los gerentes de unidad deben justificar su dominio sobre la gente que porta las competencias fundamentales de la compañía.

Elementos de la competencia funda- 
mental en óptica de Canon están esparcidos a través de negocios tan diversos como cámaras, copiadoras y equipo litográfico semiconductor y se muestran en "Competencias fundamentales de Canon". Cuando Canon identificó una oportunidad en impresoras digitales láser, esto le dio a los gerentes de SBU el derecho a reunir a otros SBU y agrupar el talento requerido. Cuando la división de productos de reprografía de Canon, se propuso desarrollar copiadoras controladas por microprocesadores, se dirigió a! grupo de productos fotográficos que había desarrollado la primera cámara controlada por microprocesador del mundo.

También los sistemas de recompensa que se centran solamente en resultados de productos de línea y los caminos profesionales que rara vez cruzan los límites de SBU, engendran patrones de conducta, entre gerentes de unidad, que son destructivamente competitivos.

En NEC, los gerentes de división se reúnen para identificar las competencias de la próxima generación. Juntos deciden cuánta inversión hay que hacer para construir cada futura competencia y la contribución en capital y personal que cada división tendrá que hacer. Hay también un sentido del intercambio equitativo. Una división puede hacer una contribución desproporcionada o recibir menos benefício del progreso logrado, pero tales inequidades de corto plazo se arreglarán a largo plazo.

Incidentalmente, la contribución positiva del gerente SBU debe hacerse visible a través de la compañia. Un administrador SBU no está dispuesto a entregar gente clave si sólo el otro negocio, (o el gerente general de ese negocio quien podría ser un competidor en la promoción) se va a beneficiar del redespliegue. Los gerentes operativos SBU deben ser alabados como jugadores de equipo. Donde las prioridades son claras, es menos probable que las transferencias se vean como indiosincráticas o motivadas políticamente.

Las Iransferencias que se hacen con el propósito de construir competercias esenciales, deben ser registradas y apreciadas en la memoria corporativa. Es razonable esperar que un negocio que ha entregado destrezas esenciales en beneficio de oportunidades corporativas, pierda en otras áreas por algún tiempo, algo de su competitividad. Si estas pérdidas en desempeño traen censura inmediata, los SBU no querrán consentir en transferir habilidades la próxima vez.

\section{La responsabilidad real de la alta gerencia es una estructura estratégica que guía la construcción de la competencia.}

Finalmente, hay maneras de desalentar a empleados claves, de la idea de que pertenecen a perpetuidad a un negocio particular. Muy pronto, en su carrera, la gente puede estar expuesta a gran variedad de negocios a través de un programa de rotación cuidadosamente planeado. En Canon, la gente crítica se mueve regularmente entre el negocio de cámaras y el de copiadoras 
y entre el negocio de copiadoras y e. de productos ópticos profesionales. A mediados de la carrera, pueden ser necesarias asignaciones periódicas a equipos interdivisionales, tanto para difundir competencias esenciales como para aflojar los lazos que pudieran atar un individuo a un negocio aun en el caso de que mejores oportunidades le hagan señas de otras partes. Aquellos que encarnan competencias esenciales críticas deberían saber que sus carreras son seguidas, guiadas por profesionales de los recursos humanos corporativos. A comienzos de los 80 , en Canon, todos los ingenieros menores de 30 años fueron invitados a inscribirse para ser miembros de un comité de siete personas que debía gastar dos años maquinando la futura dirección de Canon, incluyendo su arquitectura estratégica.

Las carreras en competencia deberian ser reunidas regularmente de todas partes de la corporación para intercambiar notas e ideas. La meta es construir un fuerte sentimiento de comunidad entre esta gente. En gran medida su lealtad debe estar con la integridad del área de competencia fundamental que ellos representan y no sólo a negocios particulares. Al viajar regularmente, hablar frecuentemente con los clientes y encontrarse con los colegas, los portadores de competencia pueden ser animados a descubrir nuevas oportunidades de mercado.

Las competencias fundamentales son la fuente del desarrollo de nuevos negocios. Ellas deben constituir el centro de la estrategia a nivel corporativo. Los administradores deben ganar liderazgo de manufactura en productos esenciales y lograr participación global a través de programas de construcción de marcas que apunten a la explotación de economías de alcance.

Sólo si la compañía se concibe como una jerarquía de competencias fundamentales, productos esenciales y unidades de negocio enfocadas en el mercado, estará lista para luchar.

La alta gerencia tampoco puede ser solamente otro estrato de consolidación contable, lo que a menudo es en un régimen de descentralización radical. La alta gerencia debe añadir mérito anunciando la arquitectura estratégica que guía el proceso de adquisición de competencia. Creemos que una obsesión por la construcción de competencia caracterizará a los ganádores globales de los 90. Con la década en camino, el tiempo para repensar el concepto de corporación ya ha pasado.

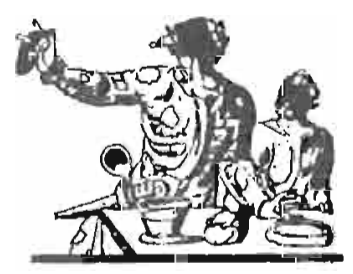




\section{Bibliografia}

\section{La calidad total y la gestión desde los agentes extemos}

Hoerr, J., "The Payoff From Teamwork", Business Week, 10 juillet 1989, p. 56-62.

Kelada, J., "La gestion integrale de la qualité", Gestion, Février 1989.

Kélada, J., Pour une qualité lotale, éditions Quafee, 1990.

Semler, R., "Managing Without Managers", Harvard Business Review, septembre-octobre 1989, p. 76-84.

Time, 7 aout 1989, p. 39.

\section{Los circulos de calidad: Lecciones de una experiencia internacional}

Audet, M., "La fonction ressources humaines a un avenir", Gestion, vol. 15, no. 1. 1990. p. 17-22.

Bank, J.K., Wilpert, B., "What's So Special About Quality Circles?". Journal of General Management, vol. 9 no. 1 1983, p. $21-27$

Barrick, M. R., Alexander, R. A., "A Review of Quality Circle Efticacy and the Existence of Positive Findings Bias". Personnel Psychology, vol. 40, 1987, p. 579-592.

Bartlett, J. B., "Success and Failure in Quality Circles: A Study of 25 Companies", Cambridge, Employment Relations Resource Center.

Beckard, R., Le développement des organisations: stratégies et modéles, Paris, Dalloz.

Blair, J. D., Ramsing, K. D., "Quality Circles and Production-Operations Management: Concerns and Caveats", Journal of Operations Management, vol. 4, no. L, 1983, p. 1-10.

Blair, J. D., Whitehead, C. J., "Can Quality Circles Survive in the United States?". Business Horizons, vol. 27, no. 5 , 1984, p. 17-23.

Bohbe, R. A., Schalfer, R. H., "Productivity Improvment: Manage it or Buy it?", Business Horizons, vol. 26, no. 2 , 1983, p. 62-69.

Bordeleau, Y., Comprendre et développer les organisations: méthodes d'analyse et d'intervention, Montréal, Agence d'Arc.

Bourbonnais, J. P., Gosselin, A., "Les défis de la gestion des ressources humaines pour les années 90: un tour d'horizon", Gestion, vol. 13, no. I, 1988, p. 23-29.

Bradley, K., Hill, S., "After Japan: the Quality Circle Transplant and Productive Efficiency", The Journal of Industrial Relations, vol. 21, no. 3, 1983, p. 291-311.

Brossard, M., "Comment assurer une plus grande longévité aux cercles de qualité"? Une voie possibte", Gestion, vol. 13, no. 4, 1988, p. [7-22.

Chevalier, F., “Des cereles de qualité á la qualité totale", Management et Qualité, vol. 25, 1988, p. 24-37.

Cole. R. E., Tachiki, D. S., "Forging Institutional Links: Making Quality Circles Work in the US", National Productivity Review, vol. 3, no. 4, 1984, p. 4l7-429

Collard, R., Dale, B., "Quality Circles: Why They Break Down and Why They Hold Up", Personnel Management vol. 17 , no. 2,1985 , p. $28-31$.

Cotton, J., Volkath, D., Froggatt, K., Lengnick-Hall, M., Jennings, K., "Employee Participation: Diverse Forms and Different Outcomes", Academy of Management Review, vol. 13, no. 1, 1988, p. 8-22.

Cresson, R., "Les cereles de qualité en France: un exemple de mise en place", Direction et Gestion, vol. I8, no. 2, 1982, p. $53-58$

Dale, B. G., "Quality Circles in the UK", Joumal of General Management, vol. 9, no. 3, 1984, p. $71-87$.

Dale, B. G., "British Quality Circles in Operation: Some Facts and Figures", Intemational Joumai of Manpower, vol. 6 , no. 4, 1985 , p. 3-10.

Dale, B., Barlow, E., "Quality Circles: The View From the Within", Management Decision, vol. 25 no. 4, 1983, p. $5-9$.

Dale, B., Hayward, S., A Sudy of Quality Circles Failures, rapport de recherche, Departmeat of Management Sciences, Institute of Science and Technology, University of Manchester, 1984a.

Dale, B., Hayward, S., "Quality Circle Failures in UK Manufacturing Companies: A Study", Omega, vol. 12, no. 5, 1984 b, p. $475-484$.

Dale, B., Hayward, S., "Quality Circle Failures in UK Manufacturing Companies: A Study, Part II", Omega, vol. 12, no. $6,1984 c$, p. $557-568$. 
Dale. B. Hayward, S., "Some of the Reasons for Qualuty Circle Falure: Part I", Leadership and Organtsation Development Journai, vol. 5, 1984 d, p. $1-6$.

Dean, J.، "The Decision to Participale in Qualily Circies", Journal of Appiied Behavioral Science, vol. 21, no. 3, = 1985, p. $317-327$.

Deppe, J., "Quality Circles in the Federal Republic of Germany", Quality Circles Journal, vol. 10, no. 2, 1987, p. $70-74$.

Donovan, M. J., "Quality Circles - Goldmine or Fall? Skillui Management of the Program Can Make the Difference", Transactions or the Fourth Anruat Conference of Internalional Association of Quality Circles, vol. 4, 1982, p. 105-111.

Drago, R., "Quality Circies: Iessons From the Lnized States", Australian Bulletin of Labour, voi. 12, no. 4, 1986, p. 244-25l.

Drago, R., "Quality Circle Survival: an Exploratory Analysis", Industrial Relations. vol. 27, no. 3, 1988, p. $336-351$

Dubori. P., "Les cerclea cie qualité: une techmque de gestion participalive efficace", Le Banquier et Revue IBC, vol. 10, no. 3, 1983, p. 36.41.

Duncan, R., McGraw, P., "Abandoning Simple Recipes agd Benelitiug From Quality Circles: An Australian Siudy", Work \& People, voi. 12, no. 2, 3986, p. 22-25.

Fertars, S., "Training For Quaisty - The Italian Experitence of Quality Circles", Journal of European Industrial Training, vol. 10, no. 3, 1986, p. 12-16.

Frazer, V., Dale, B., "UK Quality Circle Failures: The Latest Picturc", Omega, vol. 14, no. 1, 1986, p. 23-33.

Gaudreasit, C., Revue crilique de la documentation concernant les $\mathrm{CQ}$ el leurs lacteurs de réussite, mémoire de maitrise, Université de Montréal, 1986.

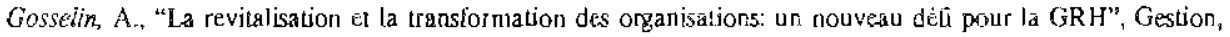
voi. 13, no. 1, $\vdots 988$. p. 36-43.

Griffin, R. W., "A iongitudinal Assessment of the Effectiveness of Quaiily Circles", Acaderny of Management Proceedings, vol. 47, 1987, p. 191-195

Griffin, R. W., "Consequences of Quality Crcies in an Industra! Setting: A Longitudinal Assessment", Academy of Management Journal, vo!. 32, no. 2, 1988, p. 338-358.

Griffin, R. W., Wayne, S. J., "A Field Stüdy of Effective and Less-Effective Quality Circles", Academy of Management Proceedicgs, vol, 44, 1984, p. 217-221.

Hernandez, J. G., Moritgomery. B., "Contributing Factors to Group Efficaey: An Exploratory Study", Transuctions of the Eigin Anrual Conference of the Intemational Association of Quality Circles, vol. 8, 1986, p. 115-123.

Hill, F. M., "Quality Circles in the UK: A Longiludirial Study", Personnel Review, vol. I5, no. 3. I986, p. $25-34$.

HadgelL5, R. M., Fountain, W. V., "The Defense Department Evaluales a Quality Circle Program", Training and Development JournaL vol. 37, no. 11, 1983, p. 98-100.

Inberman, W. "Why Quality Control Circies Don"t Work?", Canadian Business, mai 1982, p. 103-106.

Ingle, S., "How to Avoid Quality Circle Failure in your Company", Training and Development Journal, vol. 36 , no. 6,1982, n. $54-59$.

Ishikawa K., Le TQC ou ta qualité á la japoraise, Pars, Afnor Gestıon.

Jones, W. G, Velseboer, W.E. "Productivity Improvement Through Tesms: The Quality Circle A pproach", Labour and Employmemen! Gazelie, mars [985, p. 29-32.

Kahn, S., "Quality Circles in India: A Review and Assessment of the participajive Management Movement in Indian Industry", Quality Circles Journal, vol. 9, no. 3, 1986, p. 51-55.

Kelly, M., "L'analyse de contenu", in Gauthier, B., Recherche sociale, Québec, Presses de l'Université du Québec, 1984.

King, J. R., Tan, K. H.. "Quailiy Circles", Omega, vol. 14, no. 4, 1986, p. 307-315.

Lawler, E. E., Mohrman, S.A., "Quality Circles Ailer the Fad", Harvard Business Review, vol. 63, no. 1, 1985, p. $65-7 \mathrm{I}$.

Lehrer, R. N., Participative Productivity and Quality of Life Work, Englewood Clifts, Prenlice-Hal:.

Lindsay, D., "Quality Circles at Reckitt and Colman", in WVelis, C., "Quality Circles. Features of an Ausiralian Program", Work and People, vol. 8, no. 3, 1982.

Lund. R., "Industrial Democracy in Denmark", Iniemational Studies of Managemen! and Organization, vol. 17, по. 2, 1987, p. 17-26.

Marks, M. L., Hacket1, E. J.s Mirvis, P. H., Grady, J. F. Jr, "Employee Participation in Quality Circle Program: Impact on Quality of Work Life. Prodictivity and Absentesm", Journal of Appied Psycbology, vol. 71, no. 1. 1986, p. $61-69$

Menio, A. J., Steel. R. P.. "Conducling Qualily :ircles Research: Toward a Comprebersive Perspecuive", Public Productivily Review, vol. 9, 1985, p. 35-48.

Monteil' B., Alexandre, G., Ryon, P, CQ el de progtes: pour une nouvelle compélitivité, Paris, Editions d'organisation, 1983.

Mohmon, S. A., Novell, L. Jr. "Beyond Testimoniais: Learnırig From a Quality Circles Programs". Joumal of Occupational Behavior, vol. 6, 1985, p. 93-110.

Morin, P. Le développement des organisations el ia gestion des ressousces bumaines, Paris, Dunod, 1976. 
ODomell, M, ODomell. R, "Qualicy Circles. The Latest Fad or a Real Winner", Business Horizons, vol 27, no 3. 1984, p 48-52

Purtis, B., Fullerion. D J., Ingram. P R., Qualuy Circles. In Canada A Revew of Case Histones on Employee Invoivemen! Progranis, rapporl de iechercne, Schoo! or Busmess Admnistration. Univenity of Western Ontarí. 1986

Rajaet, A., "Quality Circles and Employee Alticudes". Personnd Psycholozy. vol 38, 1985. p 603-6is

Fieker. W. S., "Managenent's Role in QC". Transachons of the Second Annual Conference of the international Association of ( puality Lircies, vol. 2, 1980, p 42-46

Figny, A J, Dagnosuc organisdiorinel cas vécus, Montréai, Agence 3.Arc, 1982.

Saleh, S D., Guo, Z Hull, T "The Use of Quälity Circlés in the Automoble Parls Indistry", Actes de l'A ssociation des Sciences Adeninistrauves du Canadi, vol 9. no 5. 1988. p 95-104

Seelye. H N. Sween, J A., "Critical Components ol Successlut US Qualily Circies". The Quality Circle, Journal. vol. 6, no. I, 198,3, p $14 . ; 7$

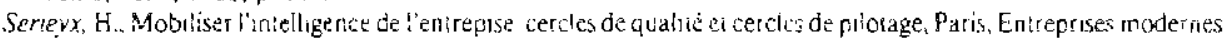
d'édilın 1982.

Seybuli, J W., Johrison, R L., "The Effectweriess or Quality Circles ar Terineco Two Years Later" rransactions of the Seventr Annual Internationa! Assoclation of Qualily Curcies Conference, vol, 7. 1985, 9 148-152.

Steet. R P., Lloyd, R. F., "Cognulve. Affectue and Behavonal Outcomes of Particspation in Qualty Circles. Conceptual and Empirica! Findings". Journa of Appled Betavional Science, vol 24. no I, 1988 , p I-I7.

Steet, R P. Mento, A J , Dilla, B L Ovalle, N K Il, Lloyd, R F. "Factors fribencing the Succets and Fallure of Two Quality Circle Programs". Jounal of Marlagemeni, vol Il no 1. 1985, p 99.119

Sieli, R. P, Shane, G.S. "Evaluation Rescarch on Quality Circles Technicai and Analyitical Implicalions" Human Reiations, vol. 39, no. 5, 1986, p 449-468

Tang. T L., Tollison, P. S, Whiteside, H, D. "She Effects of Qualizy Circle Iniriation on Motivalion To Aitenu Qualicy Circle Meetings and on Task Periormance". Personnel Psychology. vol 40, 1987, $799-814$.

Thompion. P. C., Quality Circles. How to Make Then Work in America, New York, Amacom, 1982

Turcolte, P R., Bergeron, J. L., Les ccrcles de qualite nalure el șiratégie d'implantatıon, Montréal, Agérce d'Arc, 1984.

Van Fleet, D. D. Lriffir, R W, "Quaialy Circles: A Review and Suggested Fulure Directons", in Cooper, C. L. Robertson, I. T. (éd.), International Review of Industrial and Organizational Psychology, London. Wiley, 1989.

Verma, A., McKersie, R B., "Frnployee Irvolument: The Implications of Non Imvolvinent by Unions". Ingistrial and Labor Relatrors Review, vol. 40, no. 4, 1987, p. $356-568$.

Weils, C., "Qualıy Circles. Features of and Australıan Progran". Work and People, vol. 8, no. 3, 1982, p. 12.18

Whalley. A. A., Holiman. W., "Qualny Circles Earn Union Respeci", Perionnel Journal, vol. 66, no. 12. 1987. p. $89-93$.

White, D. D., Bedrar, D A, "Localıng Problems Wuth Quality Circies", National Produclivity Revew, vui 4, no I, 1984.1985 , p. 47 .

Zahra, S. A, Lundstrom, W. J., "The Effects of Personal and Organizalıonal Chatactersucs on Participatson in Quality Circles", Akron Business and Econornic Review, sol 15, no. 2. 1984, p 33-38

\section{Modelo de simulación de gestión estratégica de Empresas Públicas GEEDAP}

Ferguson y Gould. Teoria Microeconómca. HCE. México, 1983. pp. 336-337.

Hanser Alvin. Guía de Keynes. Ed. FCE. México, 1982 pp. 90

Meier, Robert. El. A!. Téenicas de simulacion èn adminisiración y economía. Ed. Tnllas. Aléxico, 1975. pp. $32-33$.

Naylor. Thomas. el. al. Tecnicas de simulaeión én eomputadoras. Ed. Limusa. México, 1982. pp. 16-17.

Op. cit. pp. 22-23

\section{Una propuesta para la amortización de bienes de capital que maximiza el valor de la empresa}

Auerbach. Alan. Inflatıon and the Choise of Asset Life. Journal of Poltical Economy, Yol. 87, No. 3 Jure 1979. Auerbach, A. Jorgenson, D. Inflatır - Proot Deprecialion of Assecs. Harvard Business Review. Seph -Oct. 1980. Baxier, William Inthación. Efecto y Tratamienıo Contable Editoral Mc Graw Htil, México 1979

Brenner, M. Venezia, I. The Eftects of Inflation and Taxes on Growit Investments and Replacement Poltcies.

The Journat of Finance, Vol. 38 No. 5, Dec. 1983.

Clark, J.. Hindelang, T. y Pritchard, R., Capilal Budgeting Planring and Controi of Capital Expendiures. 
2da E0. Prenzice Halli, New Jeisey, 1989

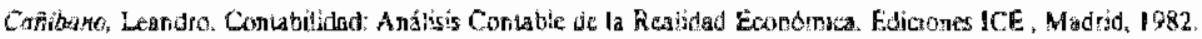

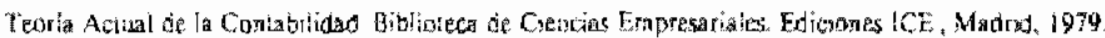

France R, Alwaré H, Pricépios de Coniabilidad para Colonbia Tipogralia Keal, Ameneila, 1908.

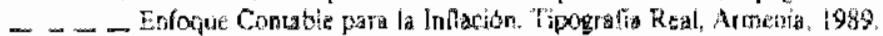

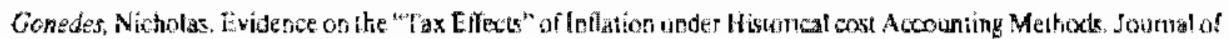
Business Vol. 54, No. 2, 1981.

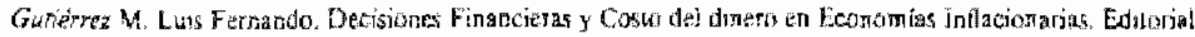
Norma, Cali 1985.

Guajardo G, Wolz P. Arler $\boldsymbol{R}_{1}$, Conlabilidad. Editotial Me Gaw Hill, Mexico 1985.

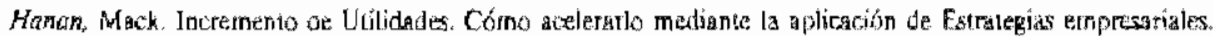
Edinorial Norma, Hegouá 1982.

Hendrcksen. Eldan. Teoria de la Contabulidad. Ediiorial Uwha, Máxico 1981.

Howe, K. Lapan, H., Inlation and Asset Lifer: The Därby verşus the Fisher Effect. Joumal of Financial and Quarijative Analysis, Vol 22, No. 2, June 1937.

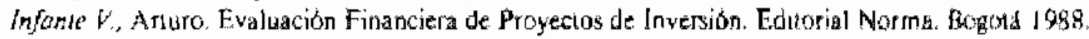

Kim, Moors. Inflationary Effects in the Capital Invesiments and Search. Journal of Business. Vol. 52, No. 3, 1979.

Lang. Theodore, Manual del Contador de Costos, Editorial Uteha, México 1973.

Lee, Cheng F.. Financial Analysis and Pianning - Theory and Application. Adjison - Weslcy Publishing

Company, Reading. Mastacluscetcls, 1985, Legrs Edicores. Ley 75 de 1986.

Muler, Martin. Guía de los P.C.G.A., Edional H.B.J. Nuevi York, 1982.

Neison, Charles. Inflatuon and Capital Budgelung. The Joumanl of Finance, Yol. 31 No. 3 June 1976.

Newman, D., Análisis Fconómico en Ingeniẹtra, Editorial Mc Grâw Hill, Méxica, 1984.

Oroziso, Guillermo A. Eloctos Financieros de los Ajustes por Inflación. Asociaciòn Bancatiá, Bogotả, 1990.

Pardinar, Felipe, Metodologia y Técnicas de Investigación en Ciencias Sociales, Editerial Siglo XXI, Rogotá, I983.

Tayior, Jorge. Ingenieria Económica, Editorial Limusa, México 1977.

Trevishick J., Mulvey Ch. Econornia de lá Inflación Editorial, El Autneo, Buenios Aires, 1975.

Venczla, I. Brenner, M. The Oplúma! Duration of Growith Invesinents ano Search. Joumal of Business,

Vol. 52. No. 3, 1979.

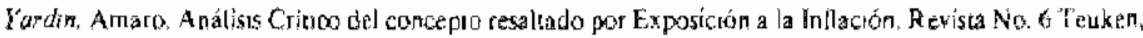
Revista de Información Contabie. Comodora, Rivadavia, Argentina 1988. 